TRANSACTIONS OF THE

AMERICAN MATHEMATICAL SOCIETY

Volume 351, Number 1, January 1999, Pages 417-428

S 0002-9947(99)02242-4

\title{
ERGODIC SEQUENCES IN THE FOURIER-STIELTJES ALGEBRA AND MEASURE ALGEBRA OF A LOCALLY COMPACT GROUP
}

\author{
ANTHONY TO-MING LAU AND VIKTOR LOSERT
}

\begin{abstract}
Let $G$ be a locally compact group. Blum and Eisenberg proved that if $G$ is abelian, then a sequence of probability measures on $G$ is strongly ergodic if and only if the sequence converges weakly to the Haar measure on the Bohr compactification of $G$. In this paper, we shall prove an extension of Blum and Eisenberg's Theorem for ergodic sequences in the Fourier-Stieltjes algebra of $G$. We shall also give an improvement to Milnes and Paterson's more recent generalization of Blum and Eisenberg's result to general locally compact groups, and we answer a question of theirs on the existence of strongly (or weakly) ergodic sequences of measures on $G$.
\end{abstract}

\section{INTRODUCTION}

Let $G$ be a locally compact group and $\pi$ be a continuous unitary representation of $G$ on a Hilbert space $H$. Let $H_{f}$ denote the fixed point set of $\pi$ in $H$, i.e.

$$
H_{f}=\{\xi \in H ; \pi(x) \xi=\xi \text { for all } x \in G\} .
$$

A sequence $\left\{\mu_{n}\right\}$ of probability measures on $G$ is called a strongly (resp. weakly) ergodic sequence if for every representation $\pi$ of $G$ on a Hilbert space $H$ and for every $\xi \in H, \quad\left\{\pi\left(\mu_{n}\right) \xi\right\}$ converges in norm (resp. weakly) to a member of $H_{f}$. When $G$ is abelian or compact, or $G$ is a [Moore]-group (i.e. every irreducible representation of $G$ is finite dimensional), then every weakly ergodic sequence is strongly ergodic. However, this is not true in general (see [8, Proposition 1 and Proposition 5]).

In [1], Blum and Eisenberg proved that if $G$ is a locally compact abelian group, and $\left\{\mu_{n}\right\}$ is a sequence of probability measures on $G$, then the following are equivalent:

(i) $\left\{\mu_{n}\right\}$ is strongly ergodic.

(ii) $\widehat{\mu}_{n}(\gamma) \rightarrow 0$ for all $\gamma \in \widehat{G} \backslash\{1\}$.

(iii) $\left\{\mu_{n}\right\}$ converges weakly to the Haar measure on the Bohr compactification of $G$.

More recently Milnes and Paterson [8] obtained the following generalization of Blum and Eisenberg's result to general locally compact groups:

Received by the editors February 3, 1997.

1991 Mathematics Subject Classification. Primary 43A05, 43A35.

Key words and phrases. Ergodic sequences, Fourier-Stieltjes algebra, measure algebra, amenable groups.

This research is supported by NSERC Grant A7679. 
Theorem A (Milnes and Paterson [8]). Let $G$ be a second countable locally compact group. Then the following statements about a sequence $\left\{\mu_{n}\right\}$ of probability measures in $M(G)$ are equivalent:

(i) $\left\{\mu_{n}\right\}$ is a weakly ergodic sequence.

(ii) $\pi\left(\mu_{n}\right) \rightarrow 0$ in the weak operator topology for every $\pi \in \widehat{G} \backslash\{1\}$.

(iii) $\widehat{\mu}_{n}$ converges to the unique invariant mean on $B_{I}(G)$, the closure in $C(G)$ of the linear span of the set of coefficient functions of the irreducible representations of $G$.

(Here $\widehat{G}$ denotes the set of irreducible continuous representations of $G$ which is the same as the dual group of $G$ when $G$ is abelian.)

Let $P_{1}(G)$ denote the continuous positive definite functions $\phi$ on $G$ such that $\phi(e)=1$ (where $e$ is the identity of $G$ ). When $G$ is abelian, $P_{1}(G)$ corresponds to the set of probability measures on the dual group $\widehat{G}$ of $G$ (by Bochner's Theorem). In this paper, we shall prove an extension of Blum and Eisenberg's Theorem for ergodic sequences in $P_{1}(G)$ (Theorems 3.1 and 3.3). We shall give an improvement to condition (iii) of Theorem A by replacing " $B_{I}(G)$ " by the Fourier-Stieltjes algebra " $B(G)$ " for any $G$ (Theorems 4.1 and 4.4) and remove the condition of second countability (= separability in [8]) in Theorem A. We shall also show that (Theorem 4.6) $G$ is $\sigma$-compact if and only if it has a strongly (or weakly) ergodic sequence of measures. This completely answers a question in [8, p. 693].

A "strongly ergodic sequence" is called a "general summing sequence" by Blum and Eisenberg in [1]. It was also introduced by Rindler under the name "unitarily distributed sequences" in Def. 4 of [13] for point sequences and their Cesàro averages and by Maxones and Rindler in [9] for sequences of measures.

\section{SOME PRELIMINARIES}

Throughout this paper, $G$ denotes a locally compact group with a fixed left Haar measure $\mu$. Integration with respect to $\mu$ will be given by $\int \cdots d x$. Let $C(G)$ denote the Banach space of bounded continuous functions on $G$ with the supremum norm. Then $G$ is amenable if there exists a positive linear functional $\phi$ on $C(G)$ of norm one such that $\phi\left(\ell_{a} f\right)=\phi(f)$ for each $a \in G$ and $f \in C(G)$ where $\left(\ell_{a} f\right)(x)=f(a x), \quad x \in G$. Amenable groups include all solvable groups and all compact groups. However, the free group on two generators is not amenable (see [11] or [12] for more details).

Let $C^{*}(G)$ denote the completion of $L^{1}(G)$ with respect to the norm $\|f\|_{c}=$ $\sup \left\{\left\|T_{f}\right\|\right\}$, where the supremum is taken over all $*$-representations $T$ of $L^{1}(G)$ as an algebra of bounded operators on a Hilbert space. Let $P(G)$ denote the subset of $C(G)$ consisting of all continuous positive definite functions on $G$, and let $B(G)$ be its linear span. Then $B(G)$ (the Fourier-Stieltjes algebra of $G$ ) can be identified with the dual of $C^{*}(G)$, and $P(G)$ is precisely the set of positive linear functionals on $C^{*}(G)$.

Let $\mathcal{B}\left(L^{2}(G)\right)$ be the algebra of bounded linear operators from $L^{2}(G)$ into $L^{2}(G)$ and let $V N(G)$ denote the weak operator topology closure of the linear span of $\{\rho(a): a \in G\}$, where $\rho(a) f(x)=f\left(a^{-1} x\right), x \in G, f \in L^{2}(G)$, in $\mathcal{B}\left(L^{2}(G)\right)$. Let $A(G)$ denote the subalgebra of $C_{0}(G)$ (continuous complex-valued functions vanishing at infinity), consisting of all functions of the form $h * \widetilde{k}$ where $h, k \in L^{2}(G)$ and $\widetilde{k}(x)=\overline{k\left(x^{-1}\right)}, x \in G$. Then each $\phi=h * \widetilde{k}$ in $A(G)$ can be regarded as an 
ultraweakly continuous functional on $V N(G)$ defined by

$$
\phi(T)=\langle T h, k\rangle \quad \text { for each } T \in V N(G) .
$$

Furthermore, as shown by Eymard in [3, pp. 210, Theorem 3.10], each ultraweakly continuous functional on $V N(G)$ is of this form. Also $A(G)$ with pointwise multiplication and the norm $\|\phi\|=\sup \{|\phi(T)|\}$, where the supremum runs through all $T \in V N(G)$ with $\|T\| \leq 1$, is a semisimple commutative Banach algebra with spectrum $G ; A(G)$ is called the Fourier algebra of $G$ and it is an ideal of $B(G)$.

There is a natural action of $A(G)$ on $V N(G)$ given by $\langle\phi \cdot T, \gamma\rangle=\langle T, \phi \cdot \gamma\rangle$ for each $\phi, \gamma \in A(G)$ and each $T \in V N(G)$. A linear functional $m$ on $V N(G)$ is called a topological invariant mean if

(i) $T \geq 0$ implies $\langle m, T\rangle \geq 0$,

(ii) $\langle m, I\rangle=1$ where $I=\rho(e)$ denotes the identity operator, and

(iii) $\langle m, \phi \cdot T\rangle=\phi(e)\langle m, T\rangle$ for $\phi \in A(G)$.

As known, $V N(G)$ always has a topological invariant mean. However $V N(G)$ has a unique topological invariant mean if and only if $G$ is discrete (see [14, Theorem 1] and [6, Corollary 4.11]).

Let $C_{\delta}^{*}(G)$ denote the norm closure of the linear span of $\{\rho(a) ; a \in G\}$. Let $B_{\delta}(G)$ denote the linear span of $P_{\delta}(G)$, where $P_{\delta}(G)$ is the pointwise closure of $A(G) \cap P(G)$. Then $B_{\delta}(G)$ can be identified with $C_{\delta}^{*}(G)^{*}$ by the map $\pi(\phi)(f)=$ $\sum\{\phi(t) f(t), t \in G\}$ for each $f \in \ell_{1}(G)$ and $\phi \in B_{\delta}(G)$ (see [3, Proposition 1.21]). Furthermore $B_{\delta}(G)$ with pointwise multiplication and dual norm is a commutative Banach algebra. If $m$ is topological invariant mean on $V N(G)$, then $m^{\prime}=$ restriction of $m$ to $C_{\delta}^{*}(G)$, is also a topological invariant mean on $C_{\delta}^{*}(G)$. Furthermore, if $m^{\prime \prime}$ is another topological invariant mean on $C_{\delta}^{*}(G)$, then $m^{\prime}=m^{\prime \prime}$, by commutativity of $B_{\delta}(G)$. If $G$ is amenable, then $B(G) \subseteq B_{\delta}(G)$. In particular, each $\phi \in B(G)$ corresponds to a continuous linear functional on $C_{\delta}^{*}(G)$ defined by $\langle\phi, \rho(a)\rangle=$ $\phi(a), a \in G$. Also if $G$ is abelian, then $C_{\delta}^{*}(G) \cong A P(\widehat{G})$, the space of continuous almost periodic functions on $\widehat{G}$ (see [5]).

\section{Some Lemmas}

Let $G$ be a locally compact group, and $M^{+}(G)$ be the positive finite regular Borel measures on $G$.

Lemma 2.1. Let $\mu \in M^{+}(G)$. For each $\phi \in A(G)$, define $S_{\phi}$ an operator on $L_{2}(G, \mu)$ by

$$
S_{\phi} h=\phi h, \quad h \in L_{2}(G, \mu) .
$$

Then the mapping $\phi \rightarrow S_{\phi}$ is a cyclic *-representation of $A(G)$ as bounded operators on $L_{2}(G, \mu)$.

Proof. It is easy to see that $\phi \rightarrow S_{\phi}$ is a $*$-representation as bounded operators on $L_{2}(G, \mu)$. Also the element $1 \in L_{2}(G, \mu)$ is a cyclic vector for $S$, since we have $\left\{S_{\phi} 1 ; \phi \in A(G)\right\}=\{\phi ; \phi \in A(G)\}$. Let $f \in C_{00}(G)$ (continuous function with compact support); then there exists $\left\{\phi_{n}\right\} \subseteq A(G)$ such that $\left\|\phi_{n}-f\right\|_{\infty} \rightarrow 0$. In particular, $\phi_{n} \rightarrow f$ in the $L_{2}$-norm of $L_{2}(G, \mu)$. The result now follows by density of $C_{00}(G)$ in $L_{2}(G, \mu)$, and $\mu(G)<\infty$. 
Lemma 2.2. Let $\{T, H\}$ be a cyclic $*$-respresentation of $A(G)$. There exists a measure $\mu \in M^{+}(G)$ such that $T$ is unitarily equivalent to a representation $S$ defined by $\mu$ as in Lemma 2.1 .

Proof. Indeed, for any $\phi \in A(G)$,

$$
\|T(\phi)\|_{\mathrm{sp}} \leq\|\phi\|_{\mathrm{sp}}
$$

$\left(\|\cdot\|_{\mathrm{sp}}\right.$ denotes the spectral-radius). Since $A(G)$ is commutative, $\|T(\phi)\|_{\mathrm{sp}}=$ operator norm in $\mathcal{B}(H)$ and $\|\phi\|_{\mathrm{sp}}=\sup \{|\phi(x)|: x \in G\}$ (by semi-simplicity of $A(G)$, and the fact that the spectrum of $A(G)$ is $G)$. Hence

$$
\|T(\phi)\| \leq\|\phi\|_{\infty} .
$$

In particular $T$ extends to a *-representation of the $C^{*}$-algebra $C_{0}(G)$ (by density of $A(G)$ in $\left.C_{0}(G)\right)$. Let $\eta \in H$ be a cyclic vector of $\{T, H\}$. Then

$$
f \rightarrow\langle T(f) \eta, \eta\rangle, \quad f \in C_{0}(G),
$$

defines a positive linear functional on the $C^{*}$-algebra $C_{0}(G)$. Let $\mu \in M^{+}(G)$ which represents this functional and $S$ be the cyclic representation of $A(G)$ as defined in Lemma 2.1. Then $T$ and $S$ are unitarily equivalent. Indeed, define a $\operatorname{map} W:\{T(\phi) \eta ; \phi \in A(G)\} \rightarrow\{\phi \cdot 1 ; \phi \in A(G)\} \subseteq L_{2}(G, \mu)$ by $W(T(\phi) \eta)=\phi \cdot 1$.

Then $\langle T(\phi) \eta, \eta\rangle=\int \phi d \mu=0$ whenever $\phi=0 \mu$-a.e. Hence $W$ is well-defined. Also

$$
\begin{aligned}
\langle T(\phi) \eta, T(\phi) \eta\rangle & =\left\langle T^{*}(\phi) T(\phi) \eta, \eta\right\rangle=\langle T(\bar{\phi} \phi) \eta, \eta\rangle \\
& =\int \bar{\phi} \phi(x) d \mu(x)=\langle\phi, \phi\rangle .
\end{aligned}
$$

Consequently $W$ extends to a linear isometry from $H$ onto $L^{2}(G, \mu)$. Finally, if $\psi, \phi \in A(G)$, then

$$
S(\phi) W(T(\psi) \eta)=S(\phi)(\psi \cdot 1)=\phi \psi \cdot 1
$$

and

$$
W T(\phi)(T(\psi) \eta)=W T(\phi \psi) \eta=\phi \psi \cdot 1
$$

Hence $\left\{S, L_{2}(G, \mu)\right\}$ and $\{T, H\}$ are unitarily equivalent.

Assume that $G$ is amenable. Then it is well known that $A(G)$ has an approximate identity bounded by 1 . Let $\{T, H\}$ be a *-representation of $A(G)$ which is nondegenerate. Next, we will show that for each $\psi \in B(G)$, there is a unique bounded linear operator $\widetilde{T}(\psi)$ on $H$ such that

(i) $\widetilde{T}(\psi) T(\phi)=T(\psi \phi)$ for all $\phi \in A(G)$.

Uniqueness is clear from the fact that vectors of the form $\{T(\phi) \xi, \phi \in A(G), \xi \in$ $H\}$ span $H$. For existence, consider first the case when $T$ is cyclic, and let $\xi_{0} \in H$ be such that $\left[T(A(G)) \xi_{0}\right]=H$. We claim that

(ii) $\left\|T(\psi \phi) \xi_{0}\right\| \leq\|\psi\|\left\|T(\phi) \xi_{0}\right\|$ for each $\phi \in A(G)$. 
Indeed, let $\phi \in A(G)$ be fixed. Choose a bounded approximate identity $\left\{\psi_{n}\right\}$ in $A(G)$ such that $\left\|\psi_{n}\right\| \leq 1$. Then

$$
\begin{aligned}
\left\|T(\psi \phi) \xi_{0}\right\| & =\lim _{n}\left\|T\left(\psi \psi_{n} \phi\right) \xi_{0}\right\| \\
& =\lim _{n}\left\|T\left(\psi \psi_{n}\right) T(\phi) \xi_{0}\right\| \\
& \leq\left\|\psi \psi_{n}\right\|\left\|T(\phi) \xi_{0}\right\| \\
& \leq\|\psi\|\left\|T(\phi) \xi_{0}\right\|
\end{aligned}
$$

(since any $*$-homomorphism from an involutive Banach algebra into a $C^{*}$-algebra is norm decreasing) as asserted.

Now it follows that the map $T(\phi) \xi_{0} \rightarrow T(\psi \phi) \xi_{0}$ (where $\phi \in A(G)$ ) extends uniquely to an operator $\widetilde{T}(\psi)$ on $\left[T(A(G)) \xi_{0}\right]=H$ having norm at most $\|\psi\|$. The relation $\widetilde{T}(\psi) T(\phi)=T(\psi \phi)$ holds on all vectors of the form $T(\theta) \xi_{0}, \quad \theta \in A(G)$, so it holds on $H$.

For a general non-degenerate $*$-representation $T$ of $A(G)$, we simply write $T=$ $\sum \bigoplus T_{\alpha}$, each $T_{\alpha}$ cyclic, and define $\widetilde{T}(\psi)=\sum \bigoplus \widetilde{T}_{\alpha}(\psi), \quad \psi \in B(G)$.

\section{ERgodic SEQUences in $B(G)$}

A sequence $\left\{\phi_{n}\right\}$ in $A(G) \cap P_{1}(G)$ is called strongly (respectively weakly) ergodic if whenever $\{T, H\}$ is a $*$-representation of $A(G), \quad \xi \in H$, the sequence $T\left(\phi_{n}\right) \xi$ converges in the norm (resp. weak) topology to a member of the fixed point set:

$$
H_{f}=\left\{\xi \in H ; T(\phi) \xi=\xi \text { for all } \phi \in A(G) \cap P_{1}(G)\right\} .
$$

Theorem 3.1. Let $G$ be a locally compact group. The following are equivalent for a sequence $\left\{\phi_{n}\right\}$ in $A(G) \cap P_{1}(G)$ :

(i) $\left\{\phi_{n}\right\}$ is strongly ergodic.

(ii) $\left\{\phi_{n}\right\}$ is weakly ergodic.

(iii) For each $g \in G, g \neq e, \phi_{n}(g) \rightarrow 0$.

(iv) For each $T \in C_{\delta}^{*}(G),\left\langle\phi_{n}, T\right\rangle \rightarrow\langle m, T\rangle$, where $m$ is the unique topological invariant mean on $C_{\delta}^{*}(G)$.

Proof. We first observe that $\langle m, \rho(g)\rangle=0$ for all $g \in G \backslash\{e\}$, and $\langle m, \rho(e)\rangle=1$. Indeed, if $\phi \in A(G) \cap P_{1}(G)$, then $\langle m, \rho(g)\rangle=\langle m, \phi \cdot \rho(g)\rangle=\langle m, \phi(g) \rho(g)\rangle=$ $\phi(g)\langle m, \rho(g)\rangle$ (note $\langle\phi \cdot \rho(g), \psi\rangle=\langle\rho(g), \phi \psi\rangle=\phi(g) \psi(g)=\phi(g)\langle\rho(g), \psi\rangle$; hence $\phi \cdot \rho(g)=\phi(g) \rho(g))$. Now if $g \neq e$, then there exists $\phi \in A(G) \cap P_{1}(G)$ such that $\phi(g) \neq 1$ so $\langle m, \rho(g)\rangle=0$. Consequently, (iii) and (iv) are equivalent.

(ii) $\Longrightarrow$ (iii). Consider, for $g \in G$ (fixed), the representation $\{T, H\}$, where $H=\mathbb{C}, \quad T(\phi) \lambda=\phi(g) \lambda$. If $g \neq e$, then $H_{f}=\{\lambda ; T(\phi) \lambda=\lambda, \phi \in A(G) \cap$ $\left.P_{1}(G)\right\}=\left\{\lambda ; \phi(g) \lambda=\lambda, \phi \in A(G) \cap P_{1}(G)\right\}=\{0\}$. Hence if $g \neq e$, then

$$
\phi_{n}(g)=T\left(\phi_{n}\right)=T\left(\phi_{n}\right) 1=\phi_{n}(g) \cdot 1 \rightarrow 0
$$

by ergodicity of the sequence $\left\{\phi_{n}\right\}$.

(iii) $\Longrightarrow$ (i). We first assume that $\{T, H\}$ is a cyclic $*$-representation of $A(G)$. By Lemma 2.2 there exists a measure $\mu \in M^{+}(G)$ such that $T$ is unitarily equivalent to a representation $S$ on $L_{2}(G, \mu)$ as in Lemma 2.1. Hence we may assume that $T=S$, and $H=L_{2}(G, \mu)$. 
Let $h \in L_{2}(G, \mu)$. Then for each $n, m$,

$$
\left\|T\left(\phi_{n}\right) h-T\left(\phi_{m}\right) h\right\|^{2}=\int\left(\phi_{n}-\phi_{m}\right)(x) h(x) \overline{\left(\phi_{n}-\phi_{m}\right)(x) h(x)} d \mu(x) .
$$

The integrand converges pointwise to " 0 " as $n, m \rightarrow \infty$, and it is dominated by the integrable function $4|h|^{2}$. Hence by the dominated convergence theorem

$$
\left\|T\left(\phi_{n}\right) h-T\left(\phi_{m}\right) h\right\|^{2} \rightarrow 0 \quad \text { as } n, m \rightarrow \infty,
$$

i.e. $\left\{T\left(\phi_{n}\right) h\right\}$ is Cauchy. Let $f$ be the limit of $T\left(\phi_{n}\right) h$ in $L_{2}(G, \mu)$. Now if $\phi \in$ $A(G) \cap P_{1}(G), \quad h \in L_{2}(G, \mu)$, then

$$
\left\|T(\phi)\left(T\left(\phi_{n}\right) h\right)-T\left(\phi_{n}\right) h\right\|^{2}=\int\left(\phi \cdot \phi_{n}-\phi_{n}\right) h \cdot \overline{\left(\phi \cdot \phi_{n}-\phi_{n}\right) \cdot h} d \mu
$$

which again converges to zero as $n \rightarrow \infty$ by the dominated convergence theorem. So $T(\phi) f=f$, i.e. $f$ is a fixed point of $\{T, H\}$.

If $\{T, H\}$ is any *-representation of $A(G)$, then $T=\left\{T_{0}, H_{0}\right\} \oplus \sum_{\alpha \in \Gamma}\left\{T_{\alpha}, H_{\alpha}\right\}$ where $\left\{T_{0}, H_{0}\right\}$ is the degenerate part of $\{T, H\}$ and $\left\{T_{\alpha}, H_{\alpha}\right\}$ is cyclic. The result follows by applying the cyclic case to each $\left\{T_{\alpha}, H_{\alpha}\right\}$ to obtain a fixed point $f_{\alpha} \in H_{\alpha}$ of $\left\{T_{\alpha}, H_{\alpha}\right\}$. Then $f=\left(f_{\alpha}\right)$ is the limit of the sequence $\left\{T\left(\phi_{n}\right) h\right\}$ in $H$, and $T(\phi) f=f$ for all $\phi \in A(G) \cap P(G)$.

Corollary 3.2. A locally compact group $G$ is first countable if and only if $A(G)$ contains an ergodic sequence.

Proof. Let $\left\{U_{n}\right\}$ be a sequence of compact symmetric neighborhoods of the identity of $G$, such that

(i) $U_{n} \downarrow\{e\}$,

(ii) $U_{n} \cdot U_{n} \subseteq U_{n-1}$.

For each $n$, let $\phi_{n}=\frac{1}{\lambda\left(U_{n}\right)}\left(1_{U_{n}} * 1_{U_{n}}\right)$. Then $\phi_{n} \in A(G) \cap P_{1}(G)$, and $\phi_{n}(g) \rightarrow$ 0 for each $g \in G(g \neq e)$. Hence $\left\{\phi_{n}\right\}$ is ergodic by Theorem 3.1. Conversely if $\left\{\phi_{n}\right\}$ is an ergodic sequence on $A(G)$, then the topology on $G$ defined by the sequence of pseudometrics $\left\{d_{n}\right\}$, where $d_{n}(x, y)=\left|\phi_{n}(x)-\phi_{n}(y)\right|$ is Hausdorff (by Theorem 3.1(iii)) and hence must agree on any compact neighbourhood of $x, x \in G$. Consequently $G$ is first countable.

For $G$ amenable, a sequence $\left\{\phi_{n}\right\}$ in $P_{1}(G)$ is called strongly (resp. weakly) ergodic if whenever $\{T, H\}$ is a non-degenerate *-representation of $A(G)$, the sequence $\widetilde{T}\left(\phi_{n}\right) \xi$ converges in norm (resp. weakly) to a member of the fixed point set:

$$
H_{f}=\left\{\xi \in H ; T(\phi) \xi=\xi \quad \text { for all } \quad \phi \in A(G) \cap P_{1}(G)\right\}
$$

where $\widetilde{T}$ is the unique extension of $T$ to $B(G)$ as defined earlier in Section 2 .

Theorem 3.3. Let $G$ be an amenable locally compact group. The following are equivalent for a sequence $\left\{\phi_{n}\right\}$ in $P_{1}(G)$ :

(i) $\left\{\phi_{n}\right\}$ is strongly ergodic.

(ii) $\left\{\phi_{n}\right\}$ is weakly ergodic.

(iii) For each $g \in G, g \neq e, \phi_{n}(g) \rightarrow 0$.

(iv) For each $T \in C_{\delta}^{*}(G),\left\langle\phi_{n}, T\right\rangle \rightarrow\langle m, T\rangle$, where $m$ is the unique topological invariant mean on $C_{\delta}^{*}(G)$. 
Proof. Note that if $m$ is a topological invariant mean on $C_{\delta}^{*}(G)$ (i.e. $\langle m, \phi \cdot T\rangle=$ $\langle m, T\rangle$ for any $\left.\phi \in P_{1}(G) \cap A(G), \quad T \in C_{\delta}^{*}(G)\right)$, then $\langle m, \psi \cdot T\rangle=\langle m, T\rangle$, for $\psi \in P_{1}(G), T \in C_{\delta}^{*}(G)$, where $\langle\psi \cdot T, \phi\rangle=\langle T, \psi \phi\rangle$, for $\phi \in A(G)$ : indeed, let $\psi_{n} \subseteq$ $P(G) \cap A(G)$ be a bounded approximate identity for $A(G)$. Then $\left\|\psi_{n} \cdot T-T\right\| \rightarrow 0$ for all $T \in U C(\widehat{G})=A(G) \cdot V N(G) \supseteq C_{\delta}^{*}(G)$. Hence $\langle m, \psi \cdot T\rangle=\lim _{n}\left\langle m, \psi \cdot \psi_{n} \cdot T\right\rangle=$ $\langle m, T\rangle$. So (iii) $\Longleftrightarrow$ (iv) as in the proof of Theorem 3.1.

(i) $\Longleftrightarrow$ (ii) $\Longleftrightarrow$ (iii): same as Theorem 3.1 (Note: the representation $T(\phi) \lambda=$ $\phi(g) \lambda$, where $\phi \in A(G)$ has a unique extension $\widetilde{T}$ to $B(G), \quad \widetilde{T}(\phi) \lambda=\phi(g) \lambda$, for $\phi \in B(G)$; similarly, the unique extension of $S$ from $A(G)$ to $B(G)$ is $S_{\phi} h=$ $\left.\phi h, \quad h \in L^{2}(G, \mu).\right)$

\section{ERgodic SEquences of MEASURES}

Let $M(G)$ denote the space of finite regular Borel measures on $G$. We put $\langle\mu, f\rangle=$ $\int_{G} f(t) d \mu(t)$, for $\mu \in M(G), f \in C(G)$ (in [8] this is denoted by $\widehat{\mu}(f)$ ). If $\pi$ is a continuous unitary representation of $G$, let $P_{f}$ denote the orthogonal projection from $H^{\pi}$ onto the closed subspace $H_{f}^{\pi}$ of fixed points.

Theorem 4.1. Let $G$ be a locally compact group. Then the following statements about a sequence $\left\{\mu_{n}\right\}$ of probability measures on $G$ are equivalent:

(i) $\left\{\mu_{n}\right\}$ is a weakly ergodic sequence.

(ii) $\pi\left(\mu_{n}\right) \rightarrow 0$ in the weak operator topology for every $\pi \in \widehat{G} \backslash\{1\}$.

(iii) $\mu_{n} \rightarrow m$ in the weak*-topology $\left(\sigma\left(B(G)^{*}, B(G)\right)\right)$, where $m$ is the unique translation-invariant mean on $B(G)$.

Proof. (i) $\Longrightarrow$ (ii). Let $\pi \in \widehat{G}$. Then $\pi\left(\mu_{n}\right) \rightarrow P_{f}$. But $P_{f}=0$ or $I$ by irreducibility of $\pi$. Hence if $\pi \neq I, \quad \pi\left(\mu_{n}\right) \rightarrow 0$ in the weak operator topology.

(ii) $\Longrightarrow$ (iii). Let $\pi \in \widehat{G}, \quad \xi, \eta \in H^{\pi}$ and $\phi_{\xi, \eta}^{\pi}(x)=\langle\pi(x) \xi, \eta\rangle, \quad x \in G$. Then

$$
\begin{aligned}
\left\langle\mu_{n}, \phi_{\xi, \eta}^{\pi}\right\rangle & =\int \phi_{\xi, \eta}^{\pi}(x) d \mu_{n}(x) \\
& =\int\langle\pi(x) \xi, \eta\rangle d \mu_{n}(x) \\
& =\left\langle\pi\left(\mu_{n}\right) \xi, \eta\right\rangle \rightarrow 0 \text { if } \pi \neq I .
\end{aligned}
$$

Let $\mathcal{E}(G)$ denote the extreme points of $P_{1}(G)$. The above implies that $\left\langle\mu_{n}, \ell_{y} \phi\right\rangle \rightarrow 0$ for any $y \in G, \phi \in \mathcal{E}(G), \quad \phi \neq 1$ where 1 denotes the constant one function on $G$. We will show that $\left\langle\mu_{n}, \phi\right\rangle \rightarrow\langle m, \phi\rangle$ for all $\phi \in P_{1}(G)$.

Note that if $E$ is a locally convex space, and $C$ a compact subset of $E$, and $f_{n}$ a sequence of continuous linear functionals on $E$ which are uniformly bounded on $C$ and converge to 0 on $C$, then convergence to 0 holds on the closed convex hull of $C$ (see [15] or [10] for an elementary proof). This applies easily if $G$ is discrete. In the general case, slight complications arise: the set $P_{1}(G)$ is not weak ${ }^{*}$-compact, and measures are in general not weak*-continuous on $B(G)$. Nevertheless the method of proof generalizes to this case:

If $G$ is second countable, then the weak*-topology on the unit ball of $B(G)$ is metrizable. Then $P_{0}(G)$ (= intersection with the cone of positive definite functions) is compact and convex; the extreme points of $P_{0}(G)$ are 0 and the extreme points of $P_{1}(G)$. Let $\phi \in P_{1}(G)$. By Choquet's theorem, there is a probability measure $\Phi$ concentrated on ext $\left(P_{0}(G)\right)$ representing $\phi$, i.e., for $T \in C^{*}(G)$, we 
have

$$
\langle T, \phi\rangle=\int_{P_{0}(G)}\langle T, \gamma\rangle d \Phi(\gamma) \text { for all } x \in G .
$$

Using a bounded approximate unit $\left(v_{n}\right)$ in $L^{1}(G) \subseteq C^{*}(G)$, it follows that the map $(x, \gamma) \rightarrow \gamma(x)=\lim \left\langle v_{n}, \ell_{x} \gamma\right\rangle$ is Borel measurable on $G \times P_{0}(G)$ and by dominated convergence that

$$
\phi(x)=\int_{P_{0}(G)} \gamma(x) d \Phi(\gamma) \text { for all } x \in G,
$$

in particular that 0 has weight zero (take $x=e$ ). Thus, $\Phi$ is concentrated on $\mathcal{E}(G)$. Hence if $\mu \in M(G)$, one gets by Fubini's theorem

$$
\langle\phi, \mu\rangle=\int_{\mathcal{E}(G)}\langle\gamma, \mu\rangle d \Phi(\gamma)
$$

Hence if $\left\{\mu_{n}\right\}$ is a sequence of probability measures on $G$, satisfying (ii), it follows from the Lebesgue dominated convergence theorem that $\left\langle\phi, \mu_{n}\right\rangle \rightarrow \Phi(\{1\})$, and similarly $\left\langle\phi, \ell_{y}^{*} \mu_{n}\right\rangle \rightarrow \Phi(\{1\})$ for $\phi \in P_{1}(G), y \in G$. Consequently, $\mu_{n}$ and $\ell_{y}^{*} \mu_{n}$ have the same limit on $P_{1}(G)$; hence $\mu_{n}$ converges to the unique invariant mean $m$ on $B(G)$.

For general $G$, if there is a weakly ergodic sequence of measures in $M(G)$ (resp. (ii) holds), then $G$ has to be $\sigma$-compact (see Theorem 4.6 and Remark 4.3).

If $G$ is $\sigma$-compact, and $\pi$ is a cyclic representation of $G$ on a Hilbert space $H$, then $H$ is separable, and hence the strong operator topology on $\mathcal{B}(H)$ is metrizable on bounded sets. Consequently, the quotient group $G / \operatorname{Ker} \pi$ is second countable, and the above argument applies.

(iii) $\Longrightarrow($ i). Let $\pi$ be a continuous unitary representation of $G$. Then, by (iii), $\left\{\left\langle\pi\left(\mu_{n}\right) \xi, \eta\right\rangle\right\}$ converges for all $\xi, \eta \in H^{\pi}$, and hence $\pi\left(\mu_{n}\right) \rightarrow T$ in the weak operator topology for some $T \in \mathcal{B}\left(H^{\pi}\right)$. Clearly, $\langle T \xi, \eta\rangle=\left\langle m, \phi_{\xi, \eta}^{\pi}\right\rangle$, and since $m$ is translation-invariant, we have $\pi(y) T=T=T \pi(y)$ for all $y \in G$. So, $T=P_{f}$ i.e. $\pi\left(\mu_{n}\right) \rightarrow P_{f}$ in the weak operator topology for all $\pi$. Hence (iii) holds.

Lemma 4.2. If $H$ is an open subgroup of $G$ with $G / H$ infinite, $\left(\mu_{n}\right)$ a weakly ergodic sequence of measures, then $\mu_{n}(H) \rightarrow 0$.

Proof. Let $\pi$ be the regular representation on $\ell^{2}(G / H), \quad \xi=1_{H}$. Then $\langle\pi(\mu) \xi, \xi\rangle=$ $\mu(H)$. If $G / H$ is infinite, it follows easily that $\ell^{2}(G / H)_{f}=(0)$; hence $\mu_{n}(H) \rightarrow$ 0 .

Remark 4.3. By a similar argument one shows that if the sequence $\left(\mu_{n}\right)$ satisfies (ii) of Theorem 4.1, then the measures $\mu_{n}$ cannot be concentrated on a subgroup $H$ as above: We have $1_{H} \in P_{1}(G)$ and the set of $\phi \in P_{1}(G)$ for which $\phi(x)=1$ for $x \in H$ is easily seen to be weak*-compact in $B(G)$. Hence it has an extreme point $\phi \neq 1$ and this is also an extreme point of $P_{1}(G)$. Thus we get $\pi \in \widehat{G} \backslash\{1\}, \quad \xi \in H^{\pi} \backslash\{0\}$ with $\pi(x) \xi=\xi$ for $x \in H$. If all $\mu_{n}$ would be concentrated on $H$, we would get $\pi\left(\mu_{n}\right) \xi=\xi$ for all $n$, contradicting (ii).

Theorem 4.4. Let $G$ be a locally compact group. Then the following statements about a sequence $\left(\mu_{n}\right)$ of probability measures on $G$ are equivalent:

(i) $\left(\mu_{n}\right)$ is a strongly ergodic sequence.

(ii) $\pi\left(\mu_{n}\right) \rightarrow 0$ in the strong operator topology for every $\pi \in \widehat{G} \backslash\{1\}$. 
Proof. (i) $\Longrightarrow$ (ii) follows as in Theorem 4.1.

(ii) $\Longrightarrow$ (i): Let $(\pi, H)$ be a (continuous, unitary) representation of $G, P_{f}$ denotes the orthogonal projection onto $H_{f}$. As in the proof of Theorem 4.1, (ii) $\Longrightarrow($ iii), we may assume that $H$ is separable, $G$ second countable. Then $C^{*}(G)$ is separable. By [16, Theorem IV.8.32] there exists a disintegration $(\pi, H)=\int_{\Gamma}^{\oplus}\left(\pi_{\gamma}, H(\gamma)\right) d \nu(\gamma)$ of the representation $(\pi, H)$ of $C^{*}(G)$ such that $\pi_{\gamma}$ is an irreducible representation of $C^{*}(G)$ for almost all $\gamma$. Each $\pi_{\gamma}$ defines a representation of $G$ and, putting $\Gamma_{f}=\{\gamma$ : $\left.\pi_{\gamma}=1\right\}$, we have clearly $H_{f}=\int_{\Gamma_{f}}^{\oplus} H(\gamma) d \nu(\gamma)$. For $\xi=\int_{\Gamma}^{\oplus} \xi(\gamma) d \nu(\gamma) \in H$, we get $P_{f} \xi=\int_{\Gamma_{f}}^{\oplus} \xi(\gamma) d \nu(\gamma)$. If $\mu$ is a bounded measure on $G$, it follows as in $[2,18.7 .4]$ that $\pi(\mu)=\int_{\Gamma}^{\oplus} \pi_{\gamma}(\mu) d \nu(\gamma)$; hence $\pi(\mu) \xi=\int_{\Gamma}^{\oplus} \pi_{\gamma}(\mu) \xi(\gamma) d \nu(\gamma)$. Since $\pi_{\gamma}\left(\mu_{n}\right) \xi(\gamma) \rightarrow 0$ for almost all $\gamma \notin \Gamma_{f}$, it follows as in the proof of Theorem 3.1, (iii) $\Longrightarrow(\mathrm{i})$, from Lebesgue's dominated convergence theorem that $\pi\left(\mu_{n}\right) \xi \rightarrow P_{f} \xi$.

Remark 4.5. The question of the existence of weakly ergodic sequences of measures was stated as a problem in [8]. In fact, the case of separable groups $G$ had already been settled before in [7], Theorem 3: for the sequences $\left(x_{n}\right)$ constructed there, $\mu_{n}=\frac{1}{n} \sum_{j=1}^{n} \delta_{x_{j}}$ has the property that $\pi\left(\mu_{n}\right)$ converges to $P_{f}$ in the strong operator topology for any continuous representation of $G$ on a Banach space $B$ for which all orbits $\{\pi(x) b: x \in G\}$ are relatively weakly compact. In particular, $\left(\mu_{n}\right)$ is even a strongly ergodic sequence. More generally, the following result holds:

Theorem 4.6. The following statements about a locally compact group $G$ are equivalent:

(i) There exists a strongly ergodic sequence of measures.

(ii) There exists a weakly ergodic sequence of measures.

(iii) $G$ is $\sigma$-compact.

Proof. (i) $\Longrightarrow$ (ii) is trivial.

(ii) $\Longrightarrow$ (iii): See Lemma 4.2 (any sequence of finite measures is supported by a countable union of compact sets, hence by an open $\sigma$-compact subgroup).

(iii) $\Longrightarrow(\mathrm{i})$ : By the Kakutani-Kodaira theorem, $G$ has a compact normal subgroup $N$ such that $G / N$ is metrizable. In particular, $G / N$ is separable. Let $\lambda$ be the normalized Haar measure on $N$ and let $M$ be a closed separable subgroup of $G$ such that $G=M \cdot N$. Let $\left(x_{n}\right)$ be a sequence in $M$ satisfying the properties of [7], Theorem 3, mentioned above. Put $\mu_{n}=\frac{1}{n} \sum_{j=1}^{n} \delta_{x_{j}} * \lambda$. We claim that $\left(\mu_{n}\right)$ is strongly ergodic. Let $(\pi, H)$ be a unitary representation of $G$. Put $H_{f, N}=\{\xi \in H: \pi(x) \xi=\xi$ for all $x \in N\}$, similarly for $H_{f, M}$, and denote the orthogonal projections on these spaces by $P_{f, N}$ resp. $P_{f, M}$. Clearly, $P_{f, N}=\pi(\lambda)$. Since $N$ is normal, $H_{f, N}$ is a $\pi$-invariant subspace; hence the same is true for $H_{f, N}^{\perp}$. This entails that $P_{f, N}$ and $P_{f, M}$ commute; hence $P_{f}=P_{f, M} \circ P_{f, N}$. By assumption, $\left(\frac{1}{n} \sum_{j=1}^{n} \pi\left(x_{n}\right)\right)$ converges strongly to $P_{f, M}$; hence $\left(\pi\left(\mu_{n}\right)\right)$ converges to $P_{f, M} \circ P_{f, N}=P_{f}$.

Examples. a) Let $H$ be the Heisenberg group. If $\left(\mu_{n}\right)$ is a sequence of probability measures, we claim that the following statements are equivalent:

(i) $\left(\mu_{n}\right)$ is strongly ergodic.

(ii) $\left(\mu_{n}\right)$ is weakly ergodic.

(iii) $\widehat{\mu}_{n}(\gamma) \rightarrow 0$ for all $\gamma \in \widehat{H} \backslash\{1\}$. 
Here $\widehat{H}$ denotes the set of abelian continuous characters of $H$, i.e. in this example strong (or weak) ergodicity is uniquely determined by the projections of $\mu_{n}$ to $H / Z$, where $Z=[H, H]$ is the center of $H$.

Proof. We use the notation of [8], Proposition 6. Condition (iii) is clearly necessary, since $\widehat{G}$ describes the one-dimensional representations of $G$. Hence it is sufficient to show that (iii) implies (i). We write $H=\mathbb{R}^{3}$ (as a set). Then the infinite dimensional irreducible representations of $H$ act on $H^{\pi}=L^{2}(\mathbb{R})$ by

$$
(\pi(x) f)(t)=e^{2 \pi i\left(x_{1}-x_{2} t\right) a} f\left(t-x_{3}\right)
$$

$\left(x=\left(x_{1}, x_{2}, x_{3}\right), a \in \mathbb{R} \backslash\{0\}\right.$ is a fixed parameter). It is clearly enough to show that $\pi\left(\mu_{n}\right) f \rightarrow 0$ for $f$ with bounded support, i.e. supp $f \subseteq[-K, K]$ for some $K>0$. Then $\langle\pi(x) f, f\rangle=0$ if $\left|x_{3}\right|>2 K$.

Put $A=\left\{(x, y) \in H \times H:\left|x_{3}-y_{3}\right| \leq 2 K\right\}$. Then it follows that $\|\pi(\mu) f\|^{2} \leq$ $\|f\|^{2} \mu \otimes \mu(A)$. Hence it is sufficient to show that

$$
\mu_{n} \otimes \mu_{n}(A) \rightarrow 0
$$

for every sequence $\left(\mu_{n}\right)$ satisfying (iii). hence

Put $\left.\left.A_{j}=\mathbb{R}^{2} \times\right] 2 K(j-1), 2 K(j+1)\right], \quad \alpha_{n j}=\mu_{n}\left(A_{j}\right)$. Then $A \subseteq \bigcup_{j \in \mathbb{Z}} A_{j} \times A_{j}$;

$$
\mu_{n} \otimes \mu_{n}(A) \leq \sum_{j} \alpha_{n j}^{2}
$$

Furthermore, $\sum_{j} \alpha_{n j} \leq 2$ (observe that $A_{j} \cap A_{k}=\phi$ for $|j-k| \geq 2$ ).

Put $\bar{\mu}_{n}(M)=\mu_{n}\left(\mathbb{R}^{2} \times M\right)$. Then $\left(\bar{\mu}_{n}\right)$ is a sequence of probability measures on $\mathbb{R}$. By assumption (iii), the sequence $\left(\bar{\mu}_{n}\right)$ converges to the Bohr-von Neumann mean $m$ on $A P(\mathbb{R})$ (we have $Z=\left\{\left(x_{1}, 0,0\right)\right\}$ ).

Fix $t \in \mathbb{N}$ with $t \geq 6$. Let $f$ be a continuous function on $\mathbb{R}$ with period $t K$, satisfying $0 \leq f \leq 1$ and

$$
f(x)=\left\{\begin{array}{lll}
1 & \text { for } \quad|x| \leq 2 K \\
0 & \text { for } \quad 3 K \leq x \leq(t-3) K
\end{array}\right.
$$

Then

$$
m(f)=\frac{1}{t K} \int_{0}^{t K} f(x) d x<\frac{6}{t} .
$$

Hence there exists $n_{0}$ such that $\left\langle f, \bar{\mu}_{n}\right\rangle<6 / t$ for $n \geq n_{0}$. Then it follows that $\alpha_{n j}<6 / t$ for $n \geq n_{0}, j=0, \pm t, \pm 2 t, \ldots$. Considering appropriate translates of $f$, we get the same estimate for the other residue classes mod $t$, i.e.

$$
\alpha_{n j}<\frac{6}{t} \quad \text { for } \quad n \geq n_{1}, \quad j \in \mathbb{Z} .
$$

This implies $\sum_{j} \alpha_{n j}^{2}<2 \cdot \frac{6}{t}$ for $n \geq n_{1}$, and for $t \rightarrow \infty$ our claim follows.

Further results of this type (in the setting of uniform distribution) have been shown in [17].

b) A similar description holds for the ' $a x+b$ '-group (compare [8], Proposition 7). In particular, a) and b) provide examples of non-Moore groups for which strong and weak ergodicity are equivalent. 
c) For $G=\mathbb{C} \underset{\sigma}{\times} \mathbb{T}$, the euclidean motion group of the plane, the situation is different. For measures $\mu_{n}$ on $G$, let as before $\bar{\mu}_{n}$ be the projections to $\mathbb{T}, m$ denotes normalized Haar measure on $\mathbb{T}$. Then we have

(i) $\left(\mu_{n}\right)$ is weakly ergodic if and only if $\bar{\mu}_{n} \rightarrow m\left(w^{*}\right)$ and $\mu_{n} \rightarrow 0$ (with respect to $\left.C_{0}(G)\right)$.

(ii) $\left(\mu_{n}\right)$ is strongly ergodic if and only if $\bar{\mu}_{n} \rightarrow m\left(w^{*}\right)$ and $\delta_{x_{n}} * \mu_{n} \rightarrow 0$ (with respect to $\left.C_{0}(G)\right)$ for arbitrary sequences $\left(x_{n}\right) \subseteq G$, i.e. the convergence $\mu_{n}(x K) \rightarrow 0$ holds uniformly for the translates of a given compact set $K$.

( $\delta_{x}$ denotes the Dirac measure concentrated at $x$.) For example, $\mu_{n}=\delta_{x_{n}} * m$, where $x_{n}$ is a sequence in $G$ tending to infinity, establishes a sequence of measures that is weakly but not strongly ergodic.

Proof. (i) follows immediately from [8], Proposition 8.

(ii) results from the following lemma. (Necessity of the condition is obvious since $\left(\mu_{n}\right)$ strongly ergodic implies $\left(\delta_{x_{n}} * \mu_{n}\right)$ strongly ergodic.)

Lemma 4.7. Let $G$ be a locally compact group, $\pi$ a unitary representation of $G$ whose coefficients $\phi_{\xi, \eta}^{\pi}$ belong to $C_{0}(G)$ and let $\left(\mu_{n}\right)$ be a sequence of probability measures on $G$ such that $\mu_{n}(x K) \rightarrow 0$ uniformly for $x \in G$ (where $K$ is a fixed compact subset of $G$ with non-empty interior). Then $\pi\left(\mu_{n}\right) \rightarrow 0$ in the strong operator topology.

Proof. Assume $\|\xi\| \leq 1$. We have

$$
\left\|\pi\left(\mu_{n}\right) \xi\right\|^{2}=\int_{G} \int_{G} \phi_{\xi, \xi}^{\pi}\left(y^{-1} x\right) d \mu_{n}(x) d \mu_{n}(y) .
$$

For $\varepsilon>0$ choose $K$ such that $\left|\phi_{\xi, \xi}^{\pi}(z)\right|<\varepsilon$ for $z \notin K$ (the condition for $\left(\mu_{n}\right)$ does not depend on the choice of $K)$. Then $\mu_{n}(y K)<\varepsilon$ for $n \geq n_{0}, y \in G$. Since $y^{-1} x \in K$ is equivalent to $x \in y K$, and $\left|\phi_{\xi, \xi}^{\pi}(z)\right| \leq 1$ for all $z$, this gives combined

$$
\left|\int_{G} \phi_{\xi, \xi}^{\pi}\left(y^{-1} x\right) d \mu_{n}(x)\right|<2 \varepsilon \quad \text { for } \quad n \geq n_{0}, y \in G .
$$

Hence $\left\|\pi\left(\mu_{n}\right) \xi\right\|^{2}<2 \varepsilon$ for $n \geq n_{0}$.

d) A similar description (as in c) but taking into account that there are no nontrivial finite dimensional unitary representations) holds for the case of non-compact, connected, simple Lie groups with finite center (compare [8], Proposition 5).

\section{REFERENCES}

[1] J. Blum and B. Eisenberg, Generalized summing sequences and the mean ergodic theorem, Proc. Amer. Math. Soc. 42 (1974), 423-429. MR 48:8749

[2] J. Dixmier, $C^{*}$-Algebras, North-Holland, Amsterdam - New York - Oxford, 1977. MR 56:16388

[3] P. Eymard, L'algèbre de Fourier d'une groupe localement compact, Bull. Soc. Math. France 92 (1964), 181-236. MR 37:4208

[4] E. Granirer and M. Leinert, On some topologies which coincide on the unit sphere of the Fourier-Stieltjes algebra $B(G)$ and of the measure algebra $M(G)$, Rocky Mountain J. Math. 11 (1981), 459-472. MR 85f:43009

[5] A.T. Lau, The second conjugate algebra of the Fourier algebra of a locally compact group, Trans. Amer. Math. Soc. 267 (1981), 53-63. MR 83e:43009

[6] A.T. Lau and V. Losert, The $C^{*}$-algebra generated by operators with compact support on a locally compact group, Journal of Functional Analysis 112 (1993), 1-30. MR 94d:22005 
[7] V. Losert and H. Rindler, Uniform distribution and the mean ergodic theorem, Inventiones Math. 50 (1978), 65-74. MR 80f:22001

[8] P. Milnes and A. Paterson, Ergodic sequences and a subspace of $B(G)$, Rocky Mountain Journal of Mathematics 18 (1988), 681-694. MR 90a:43002

[9] W. Maxones and H. Rindler, Einige Resultate ueber unitär gleichverteilte Massfolgen, Anz. Österreich Akad. Wiss., Math.-Natur. Kl. (1977/2), 11-13. MR 58:6063

[10] I. Namioka, A substitute for Lebesgue's bounded convergence theorem, Proc. Amer. Math. Soc. 12 (1961), 713-716. MR 23:A2729

[11] A.T. Paterson, Amenability, Mathematical Surveys Monographs, Vol. 29, Amer. Math. Soc. Providence, R.I., 1988. MR 90e:43001

[12] J.P. Pier, Amenable Locally Compact Groups, Wiley, New York, 1984. MR 86a:43001

[13] H. Rindler, Gleichverteilte Folgen in lokalkompakten Gruppen, Monatsh. Math. 82 (1976), 207-235. MR 55:567

[14] P.F. Renaud, Invariant means on a class of von Neumann algebras, Trans. Amer. Math. Soc. 170 (1972), 285-291. MR 46:3688

[15] H.H. Schaefer, Topological Vector Spaces, Springer-Verlag, New York-Heidelberg-Berlin, 1971. MR 49:7722

[16] M. Takesaki, Theory of Operator Algebras I, Springer, New York-Heidelberg-Berlin, 1979. MR 81e:46038

[17] K. Gröchenig, V. Losert, H. Rindler, Uniform distribution in solvable groups, Probability Measures on Groups VIII, Proceedings, Oberwolfach, Lecture Notes in Mathematics 1210, Springer, Berlin-Heidelberg-New York, 1986, pp. 97-107. MR 88d:22010

Department of Mathematical Sciences, University of Alberta, Edmonton, Alberta, Canada T6G 2G1

E-mail address: tlau@vega.math.ualberta.ca

Institut für Mathematik, Universität Wien, Strudlhofgasse 4, A-1090 Wien, Austria

E-mail address: losert@pap.univie.ac.at 